Received: 6 July 2017

Accepted: 21 August 2017

Published online: 06 September 2017

\section{Novel oscillator model with damping factor for plasmon induced transparency in waveguide} systems

\author{
Mingzhuo Zhao ${ }^{1,2}$, Hongjian $\mathrm{Li}^{1}$, Zhihui He ${ }^{1}$, Zhiquan $\mathrm{Chen}^{1}$, Hui Xu ${ }^{1}$ \& Mingfei Zheng ${ }^{1}$
}

We introduce a novel two-oscillator model with damping factor to describe the plasmon induced transparency (PIT) in a bright-dark model plasmonic waveguide system. The damping factor $\gamma$ in the model can be calculated from metal conductor damping factor $\gamma_{c}$ and dielectric damping factor $\gamma_{d}$. We investigate the influence of geometry parameters and damping factor $\gamma$ on transmission spectra as well as slow-light effects in the plasmonic waveguide system. We can find an obvious PIT phenomenon and realize a considerable slow-light effect in the double-cavities system. This work may provide guidance for optical switching and plasmon-based information processing.

Electromagnetically induced transparency (EIT) is an appealing physical phenomenon where the otherwise opaque medium becomes transparent for a probe laser modified by a coupling laser beam ${ }^{1}$, which is the elimination of absorption over a narrow spectral region in a broad absorption regime and accompanied with steep dispersion ${ }^{2,3}$. This strong dispersion plays a key role to reduce the group velocity of light ${ }^{4-7}$. But it requires complicated experimental handling because of the rather short coherence times of the superposition state. It is soon found that the characteristic features such as low absorption and steep dispersion can also be realized in classical systems such as plasmonic structures ${ }^{8-17}$. Recently, Zeng et al. reported tunable multiple phase-coupled plasmon induced transparencies in grapheme metamaterials ${ }^{18}$. Zeng et al. reported high-contrast electro-optic modulation of spatial light induced by graphene-integrated Fabry-Pérot microcavity ${ }^{19}$. Xu et al. studied the PIT transmission by a metal-insulator-metal (MIM) bus waveguide coupled with a single defective cavity based on the coupled mode theory $(\mathrm{CMT})^{20}$. He et al. reported the aspect ratio control and sensing applications for metal-dielectricmetal (MDM) slot waveguides with a multimode stub through a radiation field model (RFM ${ }^{21}$. He et al. first introduced the two-oscillator model to describe the PIT in bright-dark mode plasmonic waveguide systems ${ }^{22}$. However, the damping factor in both CMT and RFM are always first obtained from the simulation data before seeking transmission and scattering parameters.

In this paper, we approximately calculate the damping factor $\gamma$ by introducing the metal conductor damping $\gamma_{c}$ and dielectric damping $\gamma_{d}$. Transmission characteristics, slow-light effects based on coupling strength $\kappa$, geometry parameters, and damping factor $\gamma$ are discussed in detail. We find that the theoretical results are in agreement with the Finite-Difference Time-Domain (FDTD) simulations. This research may provide a new way to study PIT in the plasmonic waveguide systems and it also can give a theoretical guidance for plasmon-based information processing.

\section{Plasmonic systems and theoretical model}

We consider a metal-dielectric-metal (MDM) waveguide coupling with rectangular resonators with $\mathrm{h}=80 \mathrm{~nm}$, $\mathrm{s}_{0}=\mathrm{s}_{1}=20 \mathrm{~nm}$ as shown in Fig. 1. The metal is silver (Ag). The frequency dependent optical property of the silver nanostructure is approximated by the Drude model $\varepsilon(\omega)=\varepsilon_{\infty}-\omega_{\mathrm{p}}{ }^{2} /\left(\omega^{2}+\mathrm{i} \omega \gamma_{\mathrm{p}}\right)^{23}, \omega_{\mathrm{p}}=1.38 \times 10^{16} \mathrm{rad} / \mathrm{s}$ is the bulk plasmon frequency, $\varepsilon_{\infty}=3.7$ and $\gamma_{p}=2.37 \times 10^{13} \mathrm{rad} / \mathrm{s}$ represents the damping rate. The calculated area is divided into uniform Yee cells and surrounded by perfectly matched layer (PML) absorbing boundary. The bottom resonator is considered as the bright mode, which is directly excited by the input pulse. The top resonators which can't be directly excited by the input pulse rather than driven by the bottom resonator are considered as dark modes ${ }^{14}$.

${ }^{1}$ School of Physics and Electronic, Central South University, Changsha, 410083, PR China. ${ }^{2}$ School of Physics and Electronic, Hunan University of Science and Technology, Xiangtan, 411201, PR China. Correspondence and requests for materials should be addressed to H.L. (email: lihj398@126.com) 


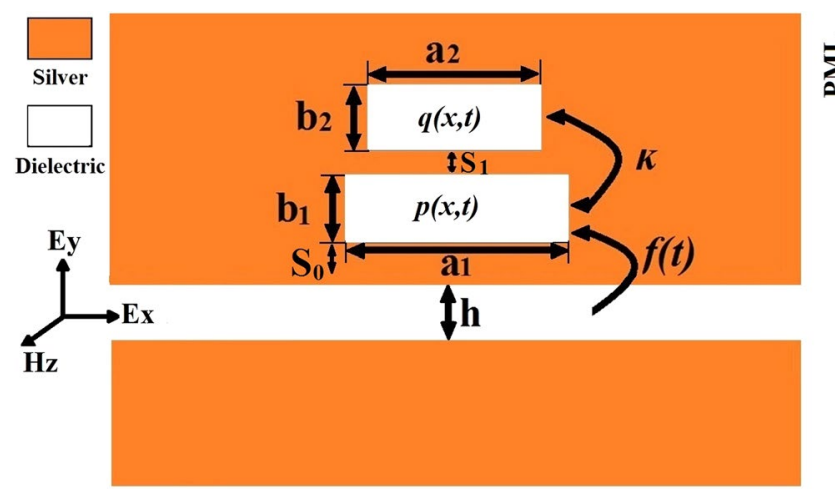

Figure 1. Schematic of a MDM waveguide side-coupled with two rectangular resonators with $\mathrm{h}=80 \mathrm{~nm}$ and $\mathrm{s}_{0}=\mathrm{s}_{1}=20 \mathrm{~nm}$.

Here, we regard the two rectangular resonators as a set of two coupled harmonic oscillators ${ }^{22}$.

$$
\begin{gathered}
\omega_{1}^{-2} \ddot{p}(x, t)+\gamma_{1} \omega_{1}^{-1} \dot{p}(x, t)+p(x, t)=f(x, t)-\kappa q(x, t) \\
\omega_{2}^{-2} \ddot{q}(x, t)+\gamma_{2} \omega_{2}^{-1} \dot{q}(x, t)+q(x, t)=-\kappa p(x, t)
\end{gathered}
$$

where damping factor $\gamma_{1}$ and $\gamma_{2}$ are described by the excitation $p(x, t)$ and $q(x, t)$, respectively. $p(x, t)$ is driven by $f(x, t)$. The two resonators are linearly coupled with coupling strength $\kappa$.

In order to investigate the rectangular resonator specifically, we introduce a method to calculate the damping factor $\gamma$, which is mainly constrained by metal conductor damping $\gamma_{c}$ and dielectric damping $\gamma_{d}$, the total damping factor is $\gamma=\gamma_{d}+\gamma_{c}$. Damping caused by metal conductor loss depends on the field distribution in the resonator, so it must be evaluated separately for each type of resonators. It is well known that each metal-dielectric interfaces supports a localized surface plasmon polaritons (SPPs) mode propagating along the $\mathrm{x}$. If the distance between the interface is comparable to or smaller than the skin depth of SPPs in dielectric, the localized modes become coupled. The coupled SPPs modes of frequency $\omega$ are described by the electromagnetic field components $U(x, y, t)=\left[E_{x}, E_{y}, H_{z}\right]$. For obtaining the intrinsic metal damping $\gamma_{c}$, it is better to use the perturbation method. The power lost per unit length due to finite wall conductivity is ${ }^{24}$

$$
P_{l}=\frac{R_{S}}{2} \oint_{C}\left|H_{z}\right|^{2} d l
$$

where $R_{s}$ is the wall surface resistance, and the integration contour $C$ encloses the inside perimeter of the resonator walls. There are surface currents on all walls.

It is instructive to compute the Poynting vector to see how power propagates in the $\mathrm{TM}_{\mathrm{m}}$ mode. According to the electromagnetic theory, the time-average power storing in a rectangular cavity is

$$
P=\frac{Z}{2} \iint_{s}\left|H_{z}\right|^{2} d S
$$

where $\mathrm{Z}$ is the wave impedance. According to electromagnetic theory and Eqs (3-4), the metal damping factor $\gamma_{c}$ in the rectangular resonators can be written in following form

$$
\gamma_{c}=\frac{P_{l}}{2 P}=\frac{R_{s}}{Z}\left(\frac{2}{b}+\frac{1}{a}\right)
$$

However, if the rectangular resonator is completely filled with a homogeneous dielectric, the damping for a lossy dielectric material can be calculated from the propagation constant, and this result will apply to any resonator with a homogeneous dielectric filling. Thus, the complex permittivity allows the complex propagation constant to be written as

$$
\begin{aligned}
\beta & =\gamma_{d}+i \alpha \\
& =\sqrt{k_{d}^{2}-k^{2}} \\
& =\sqrt{k_{d}^{2}-\omega^{2} \mu_{0} \varepsilon_{0} \varepsilon_{d}(1-j \tan \delta)}
\end{aligned}
$$

In practice, most dielectric materials have small losses (loss tangent $\tan \delta<<1$ ), and this expression can be simplified by using the first two terms of the Taylor expansion

$$
\sqrt{d^{2}+x^{2}} \approx d+\frac{1}{2}\left(\frac{x^{2}}{d}\right), \quad \text { for } \quad x \ll d
$$


Then Eq. (6) reduces to

$$
\begin{aligned}
\beta & =\sqrt{k_{d}^{2}-k^{2}+j k^{2} \tan \delta} \\
& \approx \sqrt{k_{d}^{2}-k^{2}}+\frac{j k^{2} \tan \delta}{2 \sqrt{k_{d}^{2}-k^{2}}} \\
& =\frac{k^{2} \tan \delta}{2 \alpha}+j \alpha
\end{aligned}
$$

where $\sqrt{k_{d}^{2}-k^{2}}=j \alpha$. In these results, $k=\omega \sqrt{\mu_{0} \varepsilon_{0} \varepsilon_{1}}$ is the real wave number in the absence of loss. Eq. (8) shows that the phase constant $\alpha$ is unchanged when the loss is small while the damping constant due to dielectric loss is given by

$$
\gamma_{d}=\frac{k^{2} \tan \delta}{2 \alpha}
$$

The total damping factor can be shown in the following form

$$
\gamma=\frac{R_{s}}{Z}\left(\frac{2}{b}+\frac{1}{a}\right)+\frac{k^{2} \tan \delta}{2 \alpha}
$$

In order to model scattering parameters of PIT response, electric current sheet with surface conductivity $\sigma_{s e}$ may describe this effective response. The scattering parameters of an electric current sheet are ${ }^{24}$

$$
T=\frac{2}{2+\zeta \sigma_{s e}}
$$

where $\zeta$ is the wave impedance of the external waves. We can now determine the surface conductivity from the constitutive equation

$$
\sigma_{s e} \approx \frac{-i \omega\left[1-\left(\omega / \omega_{2}\right)^{2}-i \gamma_{2}\left(\omega / \omega_{2}\right)\right]}{\left[1-\left(\omega / \omega_{1}\right)^{2}-i \gamma_{1}\left(\omega / \omega_{1}\right)\right]\left[1-\left(\omega / \omega_{2}\right)^{2}-i \gamma_{2}\left(\omega / \omega_{2}\right)\right]-\kappa^{2}}
$$

Once we have determined the surface conductivity, we can calculate the scattering parameters from Eq. (11) and other derived quantities, such as transmission phase and group index

$$
\begin{gathered}
\varphi(\omega)=\arg (T) \\
n_{g}=-\frac{1}{2} \frac{c}{L} \operatorname{Im}\left(T \frac{d \zeta \sigma_{s e}}{d \omega}\right)
\end{gathered}
$$

where $\mathrm{c}$ is the velocity of the light, and $\mathrm{L}=700 \mathrm{~nm}$ is the length of the bus waveguide.

The radiating two-oscillator model allows us to understand the response of PIT in plasmonic waveguide systems. In Fig. 2, we plot the $\gamma_{d}, \gamma_{c}$, the surface conductivity, the transmission amplitude and phase and the group index $n_{g}$ for a set of parameters. Figure 2(a) shows the relationship between $\gamma_{c}$ and geometry parameters of resonators, we can see that the $\gamma_{c}$ changes tardily with the increasing of the length and width of resonators, moreover, the value level of $\gamma_{c}$ is about $10^{-4}$. The trends of $\gamma_{d}$ as a function of the wavelength $\lambda$ and permittivity $\varepsilon$ are shown in Fig. 2(b). In Fig. 2(c-f), we recognize the typical features of PIT. The conductivity has enveloped with sharp incisions, resulting in a frequency window with large transmission at the resonance frequency. At the same time, there is large normal dispersion in the transmission phase, which leads to a significantly enhanced group delay. The reduced response can be understood from the destructive interference of the excitation due to the external field and the coupling with the dark resonator.

\section{Transmission characteristics and slow-light effects}

Coupling strength $\kappa$ is a critical factor for PIT phenomenon. It is determined by effective coupling distance, material parameters and so on. We investigate the transmission characteristics as a function of coupling strength $\kappa$ with $\kappa^{2} » \gamma_{1} \gamma_{2}^{25}$ in both theory and simulation as shown in Fig. 3(a) and (b), we can see that the theoretical transmission simulation [Fig. 3(a)] is in agreement with the FDTD simulation [Fig. 3(b)] in transmission spectrum. Figure 3(c) and (d) show the transmission phase and group index in different coupling strength $\kappa$. We find that a weak coupling strength $\kappa$ will have a big group index, so we can adjust the distance between resonant cavities to control the speed of light.

Based on the above studies, we plot the transmission spectrum of rectangular resonators in different geometry parameters of resonators as shown in Fig. 4 . The blue line shows the transmission spectrum of plasmonic waveguide systems with parameters $a_{1}=a_{2}=400 \mathrm{~nm}, b_{1}=100 \mathrm{~nm}$ and $b_{2}=80 \mathrm{~nm}$. The black line with $a_{1}=a_{2}=400 \mathrm{~nm}, b_{1}=80 \mathrm{~nm}$ and $b_{2}=100 \mathrm{~nm}$. The red line with $a_{1}=a_{2}=400 \mathrm{~nm}, b_{1}=80 \mathrm{~nm}$ and $b_{2}=80 \mathrm{~nm}$. The purple line with $b_{1}=b_{2}=80 \mathrm{~nm}, a_{1}=420 \mathrm{~nm}$ and $a_{2}=400 \mathrm{~nm}$, and the green line with $b_{1}=b_{2}=80 \mathrm{~nm}$, $\mathrm{a}_{1}=400 \mathrm{~nm}$ and $\mathrm{a}_{2}=420 \mathrm{~nm}$. By comparing the red, blue and black line, we find that the transmission spectra are almost unchanged. However, when the two rectangular resonators have the same width and the different length, the second resonance dip is red shifted by increasing the length of the bright mode resonator, and the 

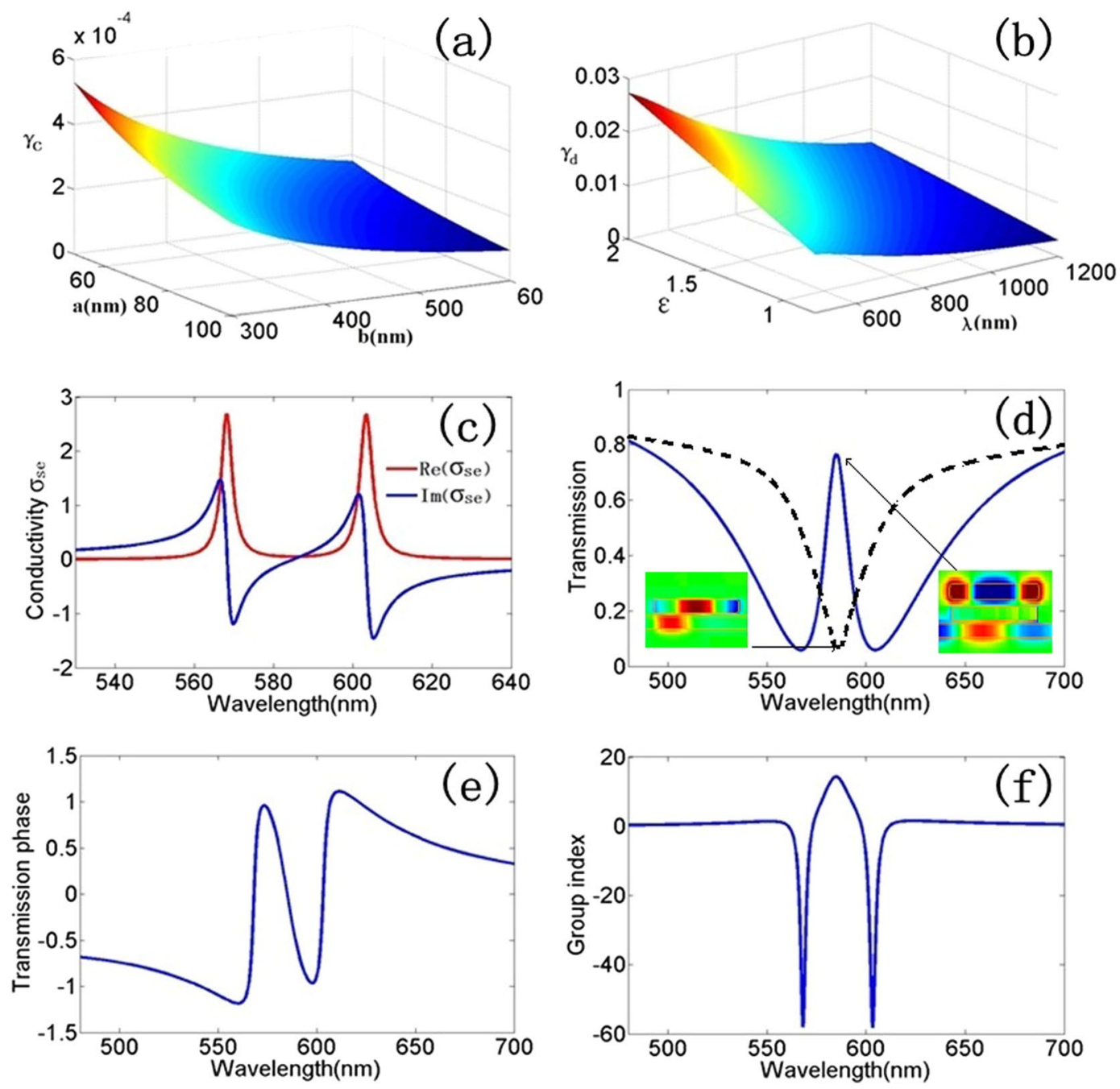

Figure 2. (a) The relationship between $\gamma_{c}$ and geometry parameters of resonators. (b) The trends of $\gamma_{d}$ due to the wavelength $\lambda$ and permittivity $\varepsilon$. (c-f) represent the spectra of surface conductivity, transmission, phase and group index of plasmonic analog of EIT as a function of wavelength in plasmonic waveguide system, respectively. Where it is described by the radiating two-oscillator mode with $\gamma_{1}=\gamma_{2}=0.006$ and $\kappa=0.06$.

first resonance dip incur red shift by increasing the length of the dark mode resonator after making a comparison between the red, purple and green lines. Based on the Eq. (5), the damping factor $\gamma_{c}$ decreases with the increasing of the width and length of rectangular resonator, but the value of the damping factor $\gamma_{c}$ is too small and the geometry parameters of resonators have little effect on damping factor $\gamma_{c}$, so the resonance wavelength of the resonators is determined by the length of the resonators.

Then, we investigate the transmission characteristics and slow-light effects as the damping factor $\gamma_{1}$ in bright mode resonator increases. In Fig. 5(a), we plot the theoretical transmission spectrum as a function of the damping factor $\gamma_{1}$ of the bright mode resonator. We can find that the transmission window becomes broad, and the peak of the transmission spectrum increases with the increasing of $\gamma_{1}$. Figure $5(b)$ and (c) show the transmission phase and group index with the increasing of the damping factor $\gamma_{1}$, we can find that a large damping factor $\gamma_{1}$ will have a large group index.

At last we research the transmission characteristics and slow-light effects of this system when the damping factor $\gamma_{2}$ in dark mode resonator increases. Figure 6(a) shows the theoretical simulation transmission spectrum as a function of the damping factor $\gamma 2$. With the damping factor increasing the transmission window of the PIT gets narrower and the transmission peak gets lower. Figure $6(\mathrm{~b})$ and $(\mathrm{c})$ show the transmission phase and group index with the increasing of the damping factor $\gamma_{2}$, and we can find that a large damping factor $\gamma_{2}$ will have a small group index. We can find an interesting phenomenon that the dark mode resonator lead to a counter transmission characteristics when the damping factor $\gamma_{2}$ increases in bright mode resonator. This phenomenon can be explained by Eqs (11-14). We can adjust the damping factor $\gamma$ of the resonant cavities to control the speed of light so that it facilitates study of photonic devices. 

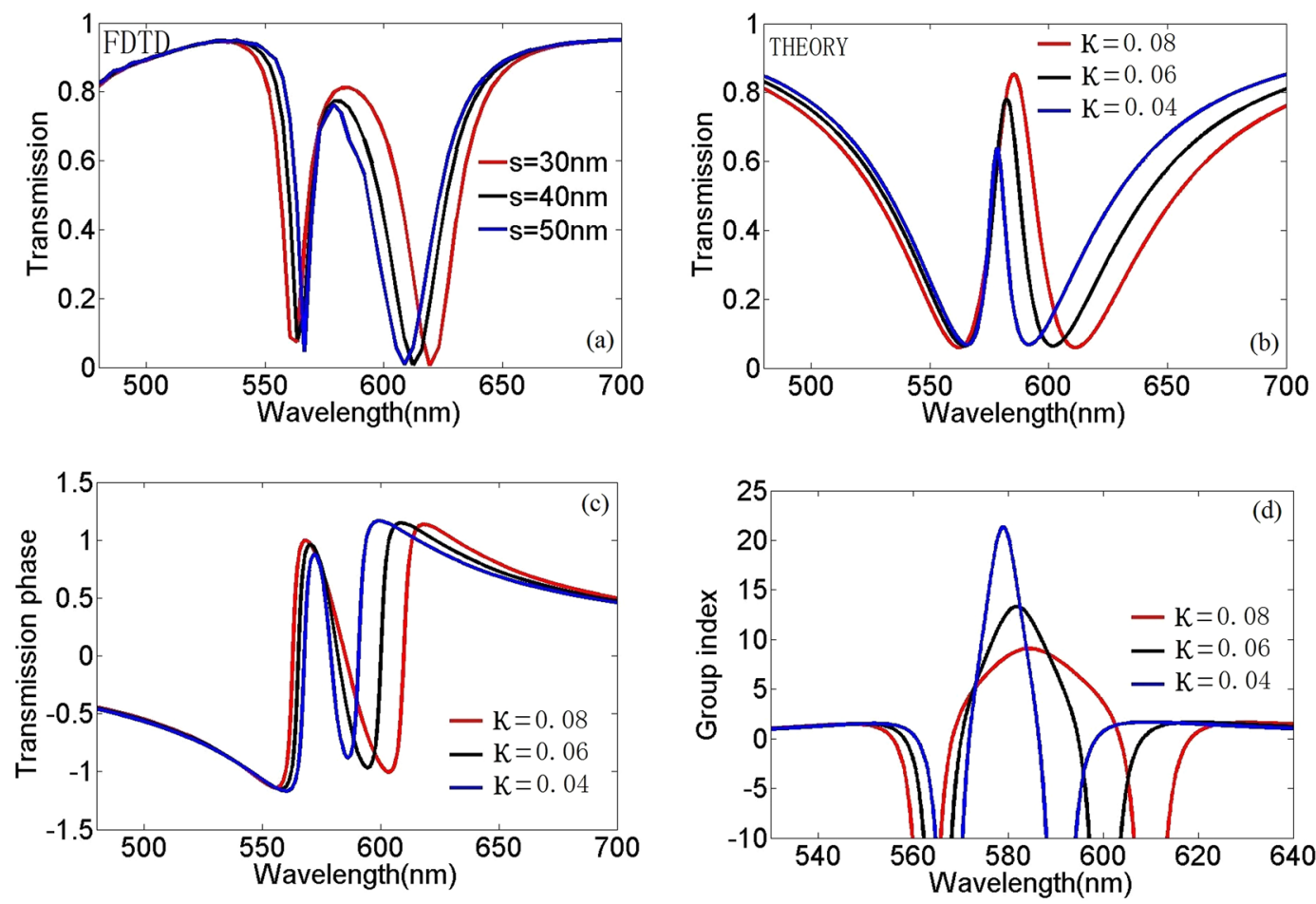

Figure 3. (a) Transmission spectra of FDTD simulations in plasmonic waveguide systems. (b) Transmission spectra of theory data in plasmonic waveguide systems. (c-d) Transmission phase and group index of the plasmonic analog of EIT in plasmonic waveguide system for different coupling strengths with $\gamma_{1}=\gamma_{2}=0.006$, respectively.

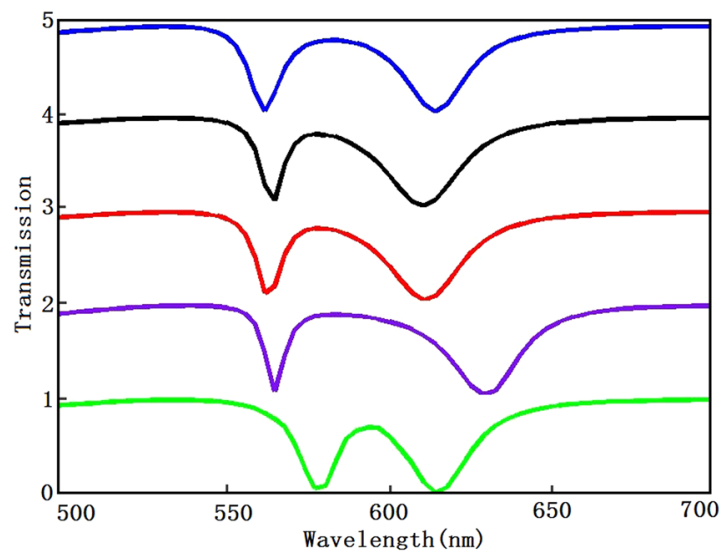

Figure 4. Transmission spectra in plasmonic waveguide systems for different geometry parameters. Blue line with parameters $a_{1}=a_{2}=400 \mathrm{~nm}, b_{1}=100 \mathrm{~nm}$ and $b_{2}=80 \mathrm{~nm}$, black line with parameters $a_{1}=a_{2}=400 \mathrm{~nm}$, $\mathrm{b}_{1}=80 \mathrm{~nm}$ and $\mathrm{b}_{2}=100 \mathrm{~nm}$, red line with parameters $\mathrm{a}_{1}=\mathrm{a}_{2}=400 \mathrm{~nm}, \mathrm{~b}_{1}=\mathrm{b}_{2}=80 \mathrm{~nm}$, purple line with parameters $b_{1}=b_{2}=80 \mathrm{~nm}, a_{1}=420 \mathrm{~nm}$ and $a_{2}=400 \mathrm{~nm}$ and green line with parameters $b_{1}=b_{2}=80 \mathrm{~nm}$, $\mathrm{a}_{1}=400 \mathrm{~nm}$ and $\mathrm{a}_{2}=420 \mathrm{~nm}$.

\section{Conclusion}

To summarize, the PIT spectral response can be realized in the bright-dark model plasmonic waveguide system. The physical mechanism of it is elucidated well by a two-oscillator model through the introduction of rigorous damping factor $\gamma$ and coupling coefficient $\kappa$. In order to investigate geometry parameters of the resonators and damping factor $\gamma$ effect on transmission characteristics and scattering parameters, we discuss the damping factor $\gamma$ through leading into the intrinsic metal damping $\gamma_{c}$ and dielectric damping $\gamma_{d}$ in detail. Our research may provide a new way to study the PIT in the plasmonic waveguide resonators. 

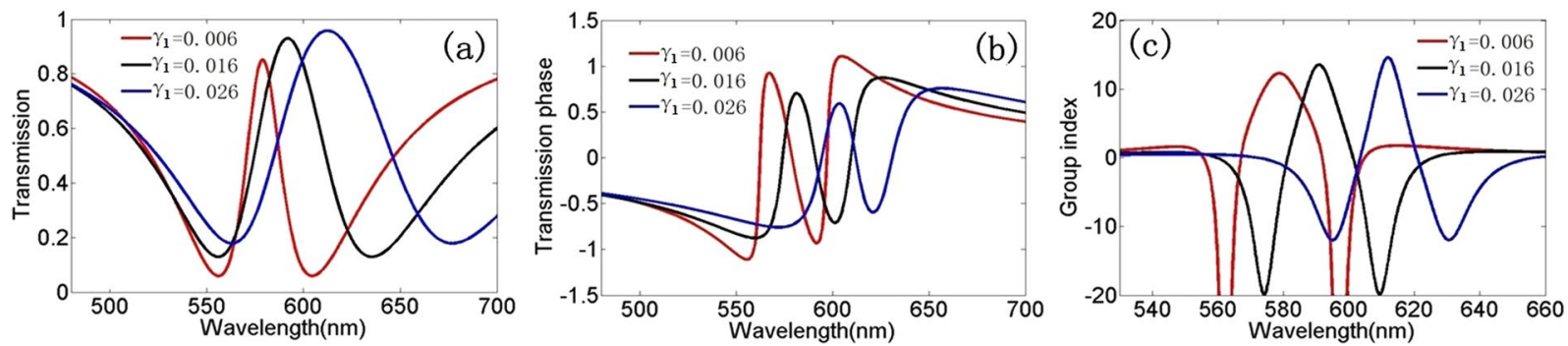

Figure 5. (a) Transmission spectra of theoretical data in plasmonic waveguide systems.(b-c). Transmission phase and group index in plasmonic waveguide systems for different $\gamma_{1}$ in superradiant mode resonator with $\kappa=0.06$ and $\gamma_{2}=0.006$.
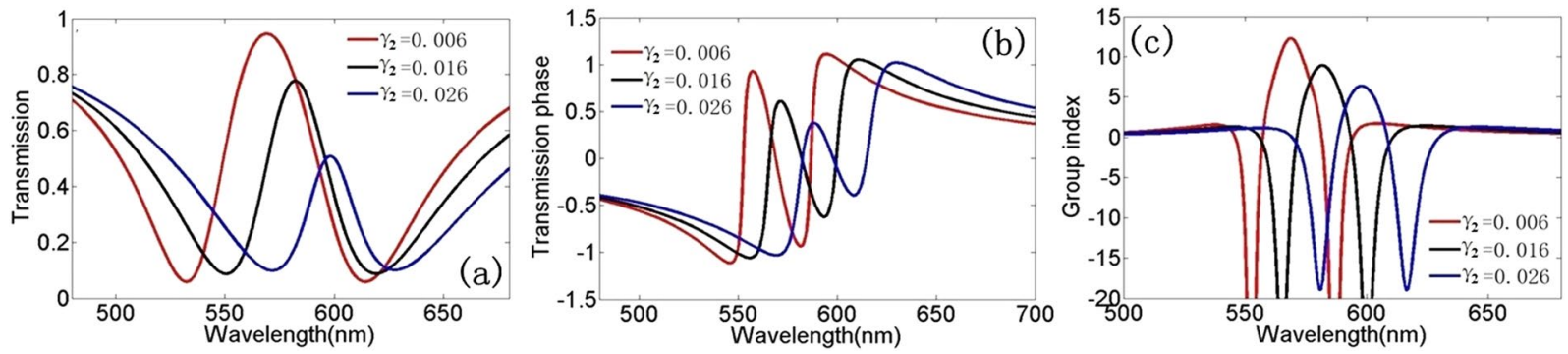

Figure 6. (a) Transmission spectrum of theoretical data in plasmonic waveguide systems. (b)-(c) Transmission phase and group index in plasmonic waveguide system for different $\gamma_{2}$ in subradiant mode resonator with $\kappa=0.06$ and $\gamma_{1}=0.006$

\section{Methods}

The frequency dependent optical property of the silver nanostructure is approximated by the Drude model $\varepsilon(\omega)=\varepsilon_{\infty}-\omega_{\mathrm{p}}{ }^{2} /\left(\omega^{2}+i \omega \gamma_{\mathrm{p}}\right)$, with $\omega_{\mathrm{p}}=1.38 \times 10^{16} \mathrm{rad} / \mathrm{s}$ is the bulk plasmon frequency, $\varepsilon_{\infty}=3.7$ and $\gamma_{p}=2.37 \times 10^{13} \mathrm{rad} / \mathrm{s}$ represents the damping rate. The characteristic spectra of the structures are found by using the two-dimensional FDTD method with mesh grid size $\Delta x=\Delta y=5 \mathrm{~nm}$. The Gauss light source is set at the entrance of the bus waveguide, and a normalized receiving screen is placed at the exit of the bus waveguide. The calculated domain is surrounded by perfectly matched layer absorbing boundary. We choose Meep as our FDTD simulation software developed by MIT. And the simulation parameters have been given in our paper.

\section{References}

1. Harris, S. E. Electromagnetically induced transparency. Phys. Today. 50, 36 (1997).

2. Boller, K. J., Imamolu, A. \& Harris, S. E. Observation of electromagnetically induced transparency. Phys. Rev. Lett. 66, 2593 (1991).

3. Fleischhauer, M., Imamoglu, A. \& Marangos, J. P. Electromagnetically induced transparency: Optics in coherent media. Rev. Mod. Phys. 77, 633 (2005).

4. Hau, L. V., Harris, S. E., Dutton, Z. \& Behroozi, C. H. Light speed reduction to $17 \mathrm{~ms}^{-1}$ in an ultracold atomic gas. Nature. 397,594 (1999).

5. Shvets, G. \& Wurtele, J. S. Transparency of magnetized plasma at the cyclotron frequency. Phys. Rev. Lett. 89, 115003 (2002).

6. Liu, C., Dutton, Z., Behroozi, C. H. \& Hau, L. V. Observation of coherent optical information storage in an atomic medium using halted light pulses. Nature. 409, 490 (2001).

7. Lukin, M. D. \& Imamoglu, A. Controlling photons using electromagnetically induced transparency. Nature. 413, 273 (2001).

8. Li, B. X., Li, H. J. \& Zeng, L. L. et al. Sensing Application in Fano Resonance With T-Shape Structure. Journal of Lightwave Technology 34(14), 3342-3347 (2016)

9. Lu, H., Liu, X., Mao, D. \& Wang, G. Plasmonic nanosensor based on Fano resonance in waveguide-coupled resonators. Opt. Lett. 37, 3780 (2012).

10. Huang, Y., Veronis, G. \& Min, C. Unidirectional reflectionless propagation in plasmonic waveguide-cavity systems at exceptional points. Opt. Express 23, 29882 (2015).

11. Huang, Y., Min, C. \& Veronis, G. Compact slit-based couplers for metal-dielectric-metal plasmonic waveguides. Opt. Express 20, 22233 (2012).

12. He, Z., Li, H. \& Li, B. et al. Theoretical analysis of ultrahigh figure of merit sensing in plasmonic waveguides with a multimode stub. Optics Letters 41(22), 5206 (2016).

13. Li, B., Li, H. \& Zeng, L. et al. Theoretical analysis and applications in inverse T-shape structure. Journal of the Optical Society of America A Optics Image Science \& Vision 33(5), 811 (2016).

14. Zhan, S. et al. Sensing analysis based on plasmon induced transparency in nanocavity-coupled waveguide. Opt. Express $\mathbf{2 3}, 20313$ (2015).

15. Chen, Z., Li, H. \& Zhan, S. et al. Tunable high quality factor in two multimode plasmonic stubs waveguide. Scientific Reports 6, 24446 (2016).

16. Cao, G. et al. Formation and evolution mechanisms of plasmon-induced transparency in MDM waveguide with two stub resonators. Opt Express 21, 9198 (2013). 
17. Zhan, S. et al. Analogy of plasmon induced transparency in detuned U-resonators coupling to MDM plasmonic waveguide. Solid State Communications. 174, 50 (2013).

18. Zeng, C., Cui, Y. \& Liu, X. Tunable multiple phase-coupled plasmoninduced transparencies in grapheme metamaterials. Optic Express 23, 545-551 (2015).

19. Zeng, C., Guo, J. \& Liu, X. High-contrast electro-optic modulation of spatial light induced by graphene-integrated fabry-pérot microcavity. Applied Physics Letters 105, 121103 (2014).

20. Xu, H., Li, H. \& Li, B. et al. Influential and theoretical analysis of nano-defect in the stub resonator. Scientific Reports 6, 30877 (2016).

21. He, Z., Peng, Y. \& Li, B. et al. Aspect ratio control and sensing applications for a slot waveguide with a multimode stub. Applied Physics Express 9(7), 072002 (2016).

22. E. D. Palik, Handbook of Optical Constants in Solids Academic (1982).

23. He, Z., Li, H. \& Zhan, S. et al. Combined theoretical analysis for plasmon-induced transparency in waveguide systems. Optics Letters 39, 5543-5546 (2014).

24. Liu, N. et al. Plasmonic analogue of electromagnetically induced transparency at the Drude damping limit. Nature material. 8, 758 (2009).

25. Tassin, P., Zhang, L., Zhao, R., Jain, A., Koschny, T. \& Soukoulis, CostasM. Electromagnetically Induced Transparency and Absorption in Metamaterials: The Radiating Two-Oscillator Model and Its Experimental Confirmation. Phys. Rev. Lett. 109, 187401 (2012).

\section{Acknowledgements}

This work was funded by the National Natural Science Foundation of China under Grant No. 61275174 and the Scientific Research Fund of Hunan Provincial Education Department under Grant No. 17C0625.

\section{Author Contributions}

This research was planned by Mingzhuo Zhao and Hongjian Li. Mingzhuo Zhao developed the analytic theory. Numberical simulation was performed by Mingzhuo Zhao, Zhihui He, Zhiquan Chen and Hui Xu. The authors Mingzhuo Zhao, Zhihui He, Zhiquan Chen, Hui Xu, and Mingfei Zheng discussed the results. Mingzhuo Zhao wrote the manuscript.

\section{Additional Information}

Competing Interests: The authors declare that they have no competing interests.

Publisher's note: Springer Nature remains neutral with regard to jurisdictional claims in published maps and institutional affiliations.

(c) (i) Open Access This article is licensed under a Creative Commons Attribution 4.0 International License, which permits use, sharing, adaptation, distribution and reproduction in any medium or format, as long as you give appropriate credit to the original author(s) and the source, provide a link to the Creative Commons license, and indicate if changes were made. The images or other third party material in this article are included in the article's Creative Commons license, unless indicated otherwise in a credit line to the material. If material is not included in the article's Creative Commons license and your intended use is not permitted by statutory regulation or exceeds the permitted use, you will need to obtain permission directly from the copyright holder. To view a copy of this license, visit http://creativecommons.org/licenses/by/4.0/.

(C) The Author(s) 2017 